\title{
Merger-triggered AGN activity and binary SMBHs in the local universe: $X$-shaped radio galaxies and double-nucleus disk galaxies
}

\section{Mezcua ${ }^{* \dagger}$}

Instituto de Astrofísica de Canarias, Vía Láctea S/N, La Laguna 38200, Tenerife, Spain

E-mail: mmezcuadiac.es

\author{
A.P. Lobanov ${ }^{1} ;$ V.H. Chavushyan ${ }^{2}$, J. León-Tavares ${ }^{3,4}$, E. Mediavilla ${ }^{5}$ \\ ${ }^{1}$ Max-Planck-Institut für Radioastronomie, Auf dem Hügel 69, 53121 Bonn, Germany \\ ${ }^{2}$ Instituto Nacional de Astrofísica, Óptica y Electrónica, Apdo. Postal 51, 72000 Puebla, México \\ ${ }^{3}$ Finnish Centre for Astronomy with ESO (FINCA), University of Turku, Väisäläntie 20, \\ FI-21500 Piikkiö, Finland \\ ${ }^{4}$ Aalto University Metsähovi Radio Observatory, Metsähovintie 114, FIN-02540 Kylmälä, \\ Finland \\ ${ }^{5}$ Instituto de Astrofísica de Canarias, Vía Láctea S/N, La Laguna 38200, Tenerife, Spain
}

\begin{abstract}
According to the hierarchical galaxy formation model, galaxies grow through mergers that can trigger AGN and starburst activity. Binary supermassive black holes (SMBH) are expected to form in the course of the merger event; however, very few pairs have been detected and the role they play in the growth of SMBHs and the triggering of AGN activity is still under debate. We present evidence of merger-triggered AGN activity at $\mathrm{z}<0.3$ from the study of the SMBH mass and starburst activity in $\mathrm{X}$-shaped radio galaxies, a type of active galaxies with a very peculiar jet morphology suggested to be the result of a merger event.

We also show that the evolution of the nuclear luminosity of double-nucleus disk galaxies can be described by a binary BH evolutionary model where the accretion disk of the secondary nucleus is being disrupted in the course of the merger event. This explains the so far low detection rate of binary SMBHs. The results also yield some potential sub-kpc AGN pair candidates.
\end{abstract}

Nuclei of Seyfert galaxies and QSOs - Central engine \& conditions of star formation, November 6-8, 2012

Max-Planck-Institut für Radioastronomie (MPIfR), Bonn, Germany

\footnotetext{
*Speaker.

${ }^{\dagger}$ Max-Planck-Institut für Radioastronomie, Auf dem Hügel 69, 53121 Bonn

¥Visiting Scientist, University of Hamburg / Deutsches Elektronen Synchrotron Forschungszentrum.
} 


\section{Introduction}

Most (if not all) galaxies host a supermassive black hole (SMBH) in their center ([37]), and the evolution of this SMBH has been found to be closely related to the formation and evolution of the host galaxy: the mass of the black hole is linked to the host galaxy through the central stellar velocity dispersion ( $M_{\mathrm{BH}^{-}} \sigma$ relationship; e.g., [16] and references therein), the bulge luminosity (e.g., [31]), and the central concentration of light (e.g., [15]), indicating that some common growth process or feedback between the SMBH and the host galaxy may exist. On the other hand it is observed that galaxies suffer frequent galaxy merging, which can enhance starburst activity and initiate/fuel AGN activity (e.g., [17]; [20]).

If nearly all galaxies host a SMBH at their center and galaxies undergo frequent galaxy merging, a pair of SMBHs is expected to be found at the center of galaxies that have recently undergone a merger (e.g., [2]; [44]). The detection and estimate of the number of binary SMBHs can thus provide important constrains on the existing models of galaxy formation and evolution, answering key questions such as: which is the galaxy and SMBH merger rate?, how much do they contribute to the SMBH growth?, or which is the role that mergers play in triggering AGNs?

Many observational evidence have been suggested in order to detect (or infer) the presence of binary SMBH systems in galaxies. In the case of spatially unresolved binary SMBHs, most of these evidence are related to peculiar radio jet morphologies like the ones in X-shaped radio galaxies (e.g., [24]; [36]) or double-double radio galaxies (e.g., [39]). Several studies have focused as well on observations of double-peaked emission line profiles (e.g., [42]; [26]), and on studies of quasar pairs (see review from [23]). Despite the numerous observational evidence that could yield the detection of a pair of SMBHs, only in a few cases have binary SMBHs been directly observed (e.g., NGC 6240, [22]; 0402+379, [38]; 3C 75, [21]).

With the aim of increasing the number of binary SMBH candidates and understanding their link to AGN-triggered activity and galaxy mergers, we have studied two type of sources suggested to harbor binary SMBH systems: X-shaped radio galaxies and double-nucleus disk galaxies.

\section{X-shaped radio galaxies}

$\mathrm{X}$-shaped radio sources owe their name to two pair of radio lobes (the high-surface-brightness and the low-surface-brightness ones) that pass symmetrically through the center of the host galaxy forming an X-shaped or winged pattern. Several scenarios were proposed to explain this peculiar radio morphology, including backflow models (e.g., [24]; [4]; [18]), reorientation of the jet axis (e.g., [8]), or a recent merger of two SMBHs (e.g., [32]; [13]; [19]). The imprints of such a merger should be reflected in the form of higher BH masses and enhanced starburst activity in the X-shaped radio galaxies when compared to a control sample of canonical double-lobed radio sources. The occurrence of a major merger event should also have led to early-type host galaxies. This scenario is tested by a multifaceted study that combines measurements of radio and optical luminosity, estimates of the $\mathrm{BH}$ masses and ages of the radio lobes, and assessments of the starburst histories in the X-shaped radio sources and in a control sample of radio-loud active nuclei ([33, 34]). 


\subsection{Sample and analysis}

The study covers a sample of $38 \mathrm{X}$-shaped radio galaxies drawn from a list of 100 candidates ([5]) and a control sample of 36 radio-loud active nuclei with similar redshift $(\mathrm{z}<0.3)$ and optical and radio luminosities. The optical spectra of the host galaxies are retrieved from the Sloan Digital Sky Survey Data Release 6 (SDSS DR6, [1]) and from spectroscopic observations carried out by [6] and [34]. The spectra are modeled using a linear combination of synthetic stellar populations with the STARLIGHT code ([7]; see also [25]), which provides the stellar velocity dispersion (from which the $\mathrm{BH}$ mass is derived using the $M_{\mathrm{BH}^{-}} \sigma$ relationship; [11]) and the light fraction, mass fraction, and metallicity of the stellar populations. From these, the starburst history of the galaxies is derived. To assess the host galaxy spectral classification of the X-shaped and control sources, the Ca II break of the absorption optical spectrum is measured and the SDSS $u, g$, and $r$-band photometry is used to locate the sources in a color-color diagram ([41]). The dynamic age of the active lobes is also estimated, from their angular size on the radio maps (see [33]).

\subsection{Results}

The host of all the $\mathrm{X}$-shaped radio galaxies studied qualify as early-type galaxies, as indicated by their Ca II break values and their location in the color-color $u$ - $g$ vs $g$ - $r$ diagram ([41]). This supports the merger hypothesis as the origin of the $\mathrm{X}$-shaped radio sources, since early-type galaxies are expected to have undergone at least one merger merger during their evolution. To compare better the properties of $\mathrm{X}$-shaped radio galaxies and the control radio sources, a sub-sample of control ellipticals that contains the 20 control sources classified as early-type galaxies by the Ca II break and the color-color diagram is defined ([33]).

A statistical study of the $\mathrm{BH}$ masses reveals that the $\mathrm{X}$-shaped radio galaxies tend to have higher BH mass than the objects in the control sample, as shown by the histograms of the BH mass distribution (Fig. 1, left). The average BH mass of the X-shaped sample is $1.93_{-0.34}^{+0.42}$ times higher than the one of the control sample in a tight common range of radio and optical luminosities. This higher mean $\mathrm{BH}$ mass could be the result of a merger event followed by a BH coalescence, which could be the cause of the $\mathrm{X}$-shaped morphology observed in the radio maps.

The most recent bursts of star formation in the X-shaped sample are found to be statistically older than those of the control sample, with $50 \%$ of the X-shaped sources having starburst ages older than $10^{8}$ years, and occurred before the active lobes were formed as indicated by the ratio of the dynamic age to the most recent starburst age ([33, 34]). Moreover, a peak at ages of 1-3 Gyr is observed in the distribution of the most recent starburst age of the X-shaped sample (Fig. 1, right). This peak suggests enhanced star formation due to a merger event, and is in agreement with the time delay between the peak of starburst activity and the end of the merger expected from simulations of galaxy mergers (e.g., [29]) and observations of individual objects (e.g., [43]; [10]). A dynamic age of $10^{6}$ yrs derived for the $\mathrm{X}$-shaped radio lobes is also in agreement with the time delay between the merger event and the onset of the radio-AGN activity triggered by the merger (e.g., [10]).

All these results strengthen the merger scenario as the proposed origin of the $\mathrm{X}$-shaped radio sources, and yield further support to the hierarchical evolutionary model in which galaxy mergers trigger star formation and AGN activity. 

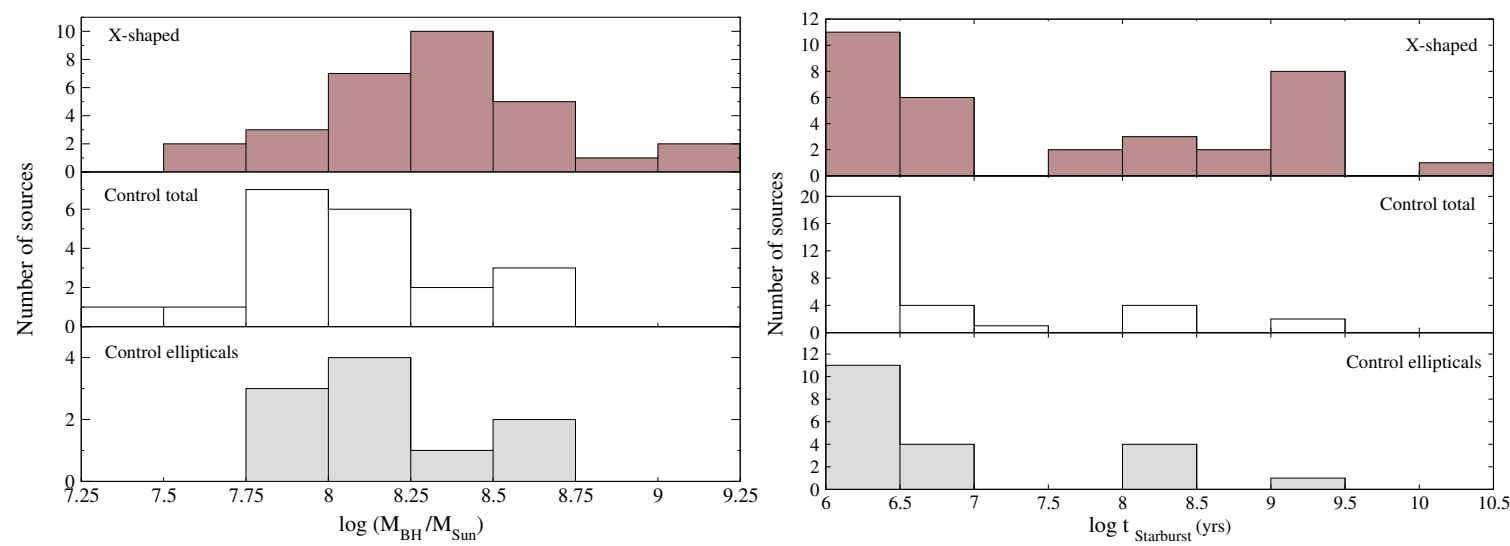

Figure 1: Histograms of the $\mathrm{BH}$ mass distribution (left) and most recent starburst age (right) for the $\mathrm{X}$-shaped sources (top), the control sample (middle), and the sub-sample of control ellipticals (bottom). Figures and caption taken from [34].

\section{Double-nucleus disk galaxies}

According to the models that describe the evolution of binary BH systems (e.g., [28] and references therein), the nuclear luminosity is expected to be affected by an enhancement of the accretion rate due to tidal effects and by accretion disk disruption. A tidal enhancement of the accretion rate is also expected from simulations of un-equal mass mergers (e.g., [3]).

We test this possible link between the evolution of a binary BH system and the strength of the nuclear activity by studying the optical luminosities of a sample of double-nucleus post-merger galaxies. A photometric PSF (Point Spread Function) fitting is used to derive the luminosity of each of the nuclei and the separation between them. We then apply a model, based on the evolution of a binary BH system ([28]), that is able to explain the observed trend between the nuclear luminosity and the relative separation between the two nuclei ([35]).

\subsection{Sample and analysis}

The sample studied consists of 54 sources drawn from a catalog of double-nucleus disk galaxies candidates of minor merger events ([14]). The sources have been selected as having optical $u, g$, and $r$-band images available in the SDSS DR8 ([9]). They all have redshift $\mathrm{z}<0.05$ and do not show strong tidal distortions indicative of major merger events ([14]).

The luminosity of each of the nuclei and their relative separation is derived from the fit of two gaussian components (PSF fitting) to the $r$-band image of the center of these double-nucleus galaxies. The code imfitfits ([30]) is used to fit a 2D photometric model (see Fig. 2), while 1D gaussian profiles are fitted using the Starlink D IP SO software package for those galaxies that could not be fitted by a 2D model ([35]).

According to the binary BH model ([27, 28]), the peak luminosity of each AGN can be described as ([35]):

$$
L_{\text {peak }}=L_{0}\left(1+\frac{\tilde{M}}{2-\tilde{M}} \frac{\tilde{M}}{\tilde{r}^{2}}\right) \xi
$$


where $L_{0}$ is the canonical luminosity of a single inactive galactic nucleus, and $\tilde{M}$ is the reduced mass of the binary system. The reduced radius $\tilde{r}$ of the system can be expressed as

$$
\tilde{r}=\frac{r}{r+r_{\mathrm{c}}},
$$

where $r_{\mathrm{c}}$ is the distance at which the two black holes become gravitationally bound and $r$ is the relative separation between the two nuclei derived from the PSF fitting. The factor $\xi$ takes into account that there is a decrease of the nuclear luminosity due to accretion disk disruption ([35]), and depends on the power-law index $\beta$ of the radial distribution of spectral intensity of the accretion $\operatorname{disk}\left(F(r) \propto r^{-\beta}\right)$.

This binary $\mathrm{BH}$ model is fitted to the observed trend between the nuclear luminosity and nuclear distance using as free parameters the distance $r_{\mathrm{c}}$, the luminosity $\mathrm{L}_{0}$, and the index $\beta$.
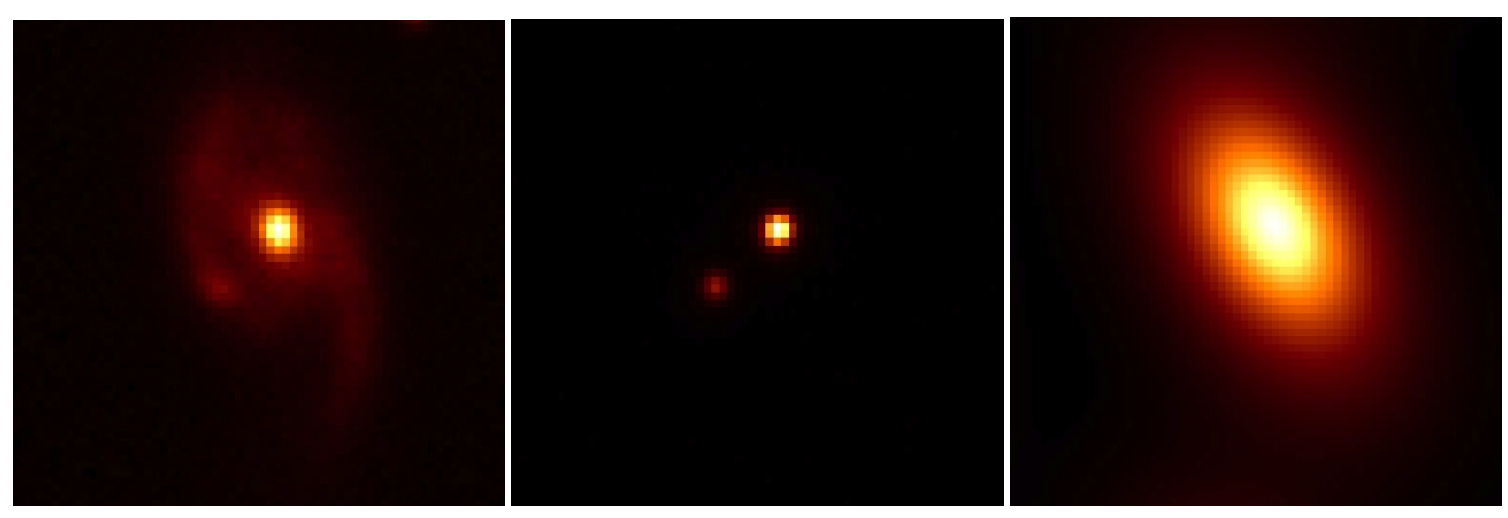

Figure 2: PSF fitting of Mrk 38. Figures from left to right show the observed SDSS $r$-band image, the model image of the 2 nuclei fitted by imfitfits, and the model image of the host galaxy fitted by imfitfits.

\subsection{Results}

The PSF fitting, the study of a connection between nuclear and host luminosity, and the fit of the binary $\mathrm{BH}$ model to the data, have yielded the following results:

- Of the 54 galaxies analyzed, 19 are found to have a double nucleus with physical separation $\leq 1 \mathrm{kpc}$.

- A trend between the nuclear luminosity of each of the nuclei and the host galaxy luminosity is observed. This trend is in agreement with the $M_{\mathrm{BH}} \propto L_{\text {bulge }}^{0.9}$ correlation derived both for inactive and active galaxies (e.g., [45]) for $r$-band luminosities $\log L_{\mathrm{R}}>8 L_{\odot}$.

- The luminosity of the secondary nucleus versus nuclear distance is satisfactorily fitted by the model, indicating that the accretion disk of the secondary nucleus is being disrupted. The value of $r_{\mathrm{c}}$ obtained from the fit indicates that the two nuclei become gravitationally bound at a projected separation of $2 \mathrm{kpc}$.

- The reduced separation, derived from the value of $r_{\mathrm{c}}$ obtained from the fit, is found to be always smaller than the distance at which the outer parts of the accretion disk of the secondary nucleus start being disrupted. This indicates that the accretion disk of the secondary nucleus has started being disrupted in all sources. 
- The value of $\beta$ obtained from the fit is used to derive the spectral index of radiation ( $\alpha$, where $F_{v} \propto v^{-\alpha}$; see review by [12]) of the accretion disk. We find a value that is in agreement with the one found in the spectral region of AGNs with a peak in the optical-UV band (the "big blue bump"; e.g., [40]), reinforcing our identification of the nuclei as SMBHs.

The study of the nuclear luminosity of these double-nucleus galaxies has proved, for the first time, the binary BH model on post-merger galaxies. The results indicate that both the tidal enhancement of the accretion rate and disk disruption are at play there, and that the accretion disk of the secondary nucleus is disrupted during a minor merger event. This indicates that the activity of the secondary BHs is rapidly quenched in post-merger galaxies, which explains the very low detection rate of binary $\mathrm{BH}$ systems.

\section{References}

[1] Adelman-McCarthy, J. K., Agüeros, M. A., Allam, S. S., et al. 2008, , 175, 297

[2] Begelman, M. C., Blandford, R. D., \& Rees, M. J. 1980, , 287, 307

[3] Callegari, S., Kazantzidis, S., Mayer, L., et al. 2011, , 729, 85

[4] Capetti, A., Zamfir, S., Rossi, P., et al. 2002, , 394, 39

[5] Cheung, C. C. 2007, , 133, 2097

[6] Cheung, C. C., Healey, S. E., Landt, H., Verdoes Kleijn, G., \& Jordán, A. 2009, , 181, 548

[7] Cid Fernandes, R., Mateus, A., Sodré, L., Stasińska, G., \& Gomes, J. M. 2005, , 358, 363

[8] Dennett-Thorpe, J., Scheuer, P. A. G., Laing, R. A., et al. 2002, , 330, 609

[9] Eisenstein, D. J., Weinberg, D. H., Agol, E., et al. 2011, , 142, 72

[10] Emonts, B. H. C., Morganti, R., Tadhunter, C. N., et al. 2006, , 454, 125

[11] Ferrarese, L., \& Merritt, D. 2000, , 539, L9

[12] Gaskell, C. M. 2008, Revista Mexicana de Astronomia y Astrofisica Conference Series, 32, 1

[13] Gergely, L. Á., \& Biermann, P. L. 2009, , 697, 1621

[14] Gimeno, G. N., Díaz, R. J., \& Carranza, G. J. 2004, , 128, 62

[15] Graham, A. W., Erwin, P., Caon, N., \& Trujillo, I. 2001, , 563, L11

[16] Graham, A. W., Onken, C. A., Athanassoula, E., \& Combes, F. 2011, , 412, 2211

[17] Hernquist, L. 1989, , 340, 687

[18] Hodges-Kluck, E. J., Reynolds, C. S., Cheung, C. C., \& Miller, M. C. 2010, , 710, 1205

[19] Hodges-Kluck, E. J., Reynolds, C. S., Miller, M. C., \& Cheung, C. C. 2010, , 717, L37

[20] Hopkins, P. F., Hernquist, L., Cox, T. J., et al. 2006, , 163, 1

[21] Hudson, D. S., Reiprich, T. H., Clarke, T. E., \& Sarazin, C. L. 2006, , 453, 433

[22] Komossa, S., Burwitz, V., Hasinger, G., et al. 2003, , 582, L15

[23] Komossa, S. 2006, , 77, 733 
[24] Leahy, J. P., \& Williams, A. G. 1984, , 210, 929

[25] León-Tavares, J., Valtaoja, E., Chavushyan, V. H., et al. 2011, , 411, 1127

[26] Liu, X., Shen, Y., Strauss, M. A., \& Greene, J. E. 2010, , 708, 427

[27] Lobanov, A. 2007, From Planets to Dark Energy: the Modern Radio Universe,

[28] Lobanov, A. P. 2008, , 79, 1306

[29] Lotz, J. M., Jonsson, P., Cox, T. J., \& Primack, J. R. 2008, , 391, 1137

[30] McLeod, B. A., Bernstein, G. M., Rieke, M. J., \& Weedman, D. W. 1998, , 115, 1377

[31] McLure, R. J., \& Dunlop, J. S. 2002, , 331, 795

[32] Merritt, D., \& Ekers, R. D. 2002, Science, 297, 1310

[33] Mezcua, M., Lobanov, A. P., Chavushyan, V. H., \& León-Tavares, J. 2011, , 527, A38

[34] Mezcua, M., Chavushyan, V. H., Lobanov, A. P., \& León-Tavares, J. 2012, , 544, A36

[35] Mezcua, M., Lobanov, A. P., Mediavilla, E., in prep.

[36] Parma, P., Ekers, R. D., \& Fanti, R. 1985, , 59, 511

[37] Richstone, D., Ajhar, E. A., Bender, R., et al. 1998, , 395, A14

[38] Rodriguez, C., Taylor, G. B., Zavala, R. T., et al. 2006, , 646, 49

[39] Schoenmakers, A. P., de Bruyn, A. G., Röttgering, H. J. A., van der Laan, H., \& Kaiser, C. R. 2000, , 315, 371

[40] Shang, Z., Brotherton, M. S., Green, R. F., et al. 2005, , 619, 41

[41] Strateva, I., Ivezić, Ž., Knapp, G. R., et al. 2001, , 122, 1861

[42] Strateva, I. V., Strauss, M. A., Hao, L., et al. 2003, , 126, 1720

[43] Tadhunter, C., Robinson, T. G., González Delgado, R. M., Wills, K., \& Morganti, R. 2005, , 356, 480

[44] Van Wassenhove, S., Volonteri, M., Mayer, L., et al. 2012, , 748, L7

[45] Wandel, A. 2002, , 565, 762 